\title{
HIV Incidence Among Non-Pregnant Women Living in Selected Rural, Semi-Rural and Urban Areas in Kwazulu-Natal, South Africa
}

\author{
Gita Ramjee · Handan Wand • Claire Whitaker • \\ Sheena McCormack • Nancy Padian • \\ Cliff Kelly $\cdot$ Andrew Nunn
}

Published online: 25 September 2011

(C) The Author(s) 2011. This article is published with open access at Springerlink.com

\begin{abstract}
The province of KwaZulu-Natal has the highest prevalence of HIV in South Africa, particularly among young women. In order to more closely examine the HIV prevalence and incidence in non-pregnant women from rural, semi-rural and urban areas, data from 5,753 women screened for enrolment into three HIV prevention studies were combined and analysed. The prevalence of HIV infection was $43 \%$ at screening. HIV incidence among the 2,523 enrolled HIV-negative women was determined every quarter, and sexual behaviour and socio-demographic data were collected as per respective protocols. During followup, 211 women seroconverted (6.6/100 women years). Multivariate analysis found that seroconversion rates were highest among women who were $\leq 24$ years old, single and not cohabiting, and who had incident sexually transmitted
\end{abstract}

G. Ramjee $(\bowtie) \cdot C$. Whitaker

HIV Prevention Research Unit, Medical Research Council, 123 Jan Hofmeyr Road, Westville, Durban 3630, South Africa e-mail: gita.ramjee@mrc.ac.za

H. Wand

National Center for HIV Epidemiology and Clinical Research, Sydney, Australia

S. McCormack · A. Nunn

Medical Research Council Clinical Trials Unit, London, UK

\section{N. Padian}

Women's Global Health Imperative, RTI International,

San Francisco, CA, USA

N. Padian

School of Public Health, University of California,

Berkeley, CA, USA

C. Kelly

Fred Hutchinson Cancer Center, Seattle, WA, USA infections. The epidemic in KwaZulu-Natal calls for targeted HIV prevention interventions among those at highest risk of acquiring or transmitting infection.

Keywords HIV - Women - Cohabitation - Risk factors · South Africa

\section{Introduction}

Sub-Saharan Africa is home to more than $60 \%$ of the total number of people living with HIV/AIDS [1]. Many countries in this region are said to have a "generalized epidemic" defined as having HIV prevalence rates of more than $1 \%$ in the adult population aged between 15-59 years and of more than $5 \%$ among populations at high risk (such as those with sexually transmitted infections (STIs)) [2].

The dominant mode of HIV transmission in the region is unprotected heterosexual sex. Factors which exacerbate the risk of HIV transmission are transactional sex [3, 4], multiple concurrent partnerships $[5,6]$, co-infection with viral and bacterial STIs [7, 8], lack of male circumcision [9, 10], and migration [11-13]. Age and gender are also important predictors of vulnerability to HIV infection, with younger women more vulnerable to HIV infection than men of the same age [14].

Unfortunately, the HIV epidemic continues to affect South Africa, and particularly South African women, disproportionately. The province of KwaZulu-Natal (KZN) is home to 9.4 million people. HIV prevalence in KZN increased by $8.6 \%$ between 2002 and 2008 to $23.5 \%$ [14], and the province has an antenatal HIV prevalence of nearly $40 \%$ [15]. HIV prevalence rates have always been higher in KZN than in any other province in South Africa, and this trend has been sustained since the early 1990s. This region 
is therefore considered to be the epicentre of the HIV/AIDS epidemic in South Africa, the area most in need of an effective HIV prevention method for women [16].

Previously, the evidence has suggested that HIV prevalence was generally higher in large urban areas compared with rural areas-residents of urban informal areas were found to have an HIV prevalence of $25.8 \%$, compared with a prevalence of $13.9 \%$ for residents of both rural and urban formal areas, and $17.3 \%$ for those residing in rural informal areas [17]. However, residents of rural areas may have less access to HIV testing, care and treatment than their urban counterparts. Additional challenges for rural communities may include poverty, lack of education, lack of access to condoms and unemployment. These factors together with cultural norms and values could influence the ability to achieve correct and consistent use of male and female condoms, the only widely available prevention technologies; this may lead to variations in HIV prevalence linked to the socio-economic profile and geographical location of communities.

Despite intensive condom counselling, many women in southern Africa are unable to negotiate safe sex [16]. Accordingly, there has been growing interest in femaleinitiated methods of HIV prevention. In recent years, research has focused on topical microbicides, products which can be discreetly applied inside the vagina before sexual intercourse. In addition, barrier methods such as the vaginal diaphragm have been tested for HIV prevention [18]. Recently, proof of concept was provided by the favourable results of the CAPRISA 004 trial of an antiretroviral-based microbicide [19], after many years of discouraging trial outcomes-six other potential microbicides and the vaginal diaphragm failed to prevent HIV infection [18, 20-25].

We conducted two site preparedness studies for future large scale HIV prevention trials, and an HIV prevention phase III clinical trial of the vaginal diaphragm in cohorts of women drawn from rural, semi-rural and urban communities in KZN. Given that women participating in such studies are requested to avoid pregnancy due to the unknown teratogenic effects of the study products, these investigations provided important information on the status of the epidemic among non-pregnant women, a demographic obviously excluded from antenatal surveys. Data from these three studies have been combined to describe the risk factors associated with HIV acquisition overall, and to determine whether prevalence and incidence differed by residential area. Direct HIV incidence measures are of interest given that these are rare, difficult to obtain, and are becoming increasingly important for the opportunity they provide to assess the impact of changes in HIV prevention programmes on seroconversion patterns. Although BED capture enzyme immunoassay
(BED-CEIA) [26] and avidity index (AI) [27] assays have been developed to estimate the incidence of HIV from samples collected in cross-sectional surveys, the accuracy of these remains to be optimised-BED-CEIA and AI tend to over-estimate incidence rates [28-30], while serial HIV prevalence surveys from which incidence may be calculated are expensive and suffer from limitations in representativeness and difficulties associated with calculation [31]. This combined dataset therefore provides a unique opportunity for more specific measurement of HIV incidence and associated risk factors in one of the most severely affected areas in the world.

\section{Methods}

\section{Study Sites}

We combined data from 5,753 sexually active women who consented to screening for three studies in KwaZuluNatal, South Africa. These studies were as follows: Methods for Improving Reproductive Health in Africa (MIRA) trial of the diaphragm for HIV prevention ( $n=3,492$; September 2002-September 2005, undertaken in rural Umkomaas and Botha's Hill, southern and western Durban, respectively) [18]; The Microbicides Development Programme (MDP) Feasibility Study in Preparation for Phase III Microbicide Trials $(n=1,226$; August 2002-September 2004, semi-rural Tongaat and Verulam, northern Durban) [32], and the HIV Prevention Trials Network (HPTN) Site Preparedness study for Future Implementation of Phase II/IIb/III clinical trials ( $n=1,035$; May 2003-January 2005, rural district of Hlabisa, and urban centre in Durban) [16]. Results from the MIRA trial indicated that use of the diaphragm did not provide added protection against HIV over that provided by condoms and an HIV prevention package [18]; we were thus able to combine the data from this trial with data from the cohort studies. The decision on the allocation of a given study site to the categories of "urban", "rural" or "semi-rural" was based upon the relative population density and level of infrastructural development of the communities surrounding each site. Thus Umkomaas, Botha's Hill and Hlabisa were considered to be "rural" communities given the lower population density, minimal infrastructure and prevalence of subsistence agricultural activities, while Verulam and Tongaat were considered to be intermediate between these communities and an urban area, since both of these towns provide commercial and retail services to a wider population residing in surrounding areas which are relatively less developed, resulting in an overall intermediate population mix for the clinics in Verulam and Tongaat. 
Population

All study populations have previously been described elsewhere [16, 18, 32]. Women were recruited from family planning, well-baby and general health clinics for the MIRA study; in the HPTN 055 study, women were recruited through community meetings and door-to-door visits; while the MDP Feasibility study recruited women from health centres, family planning clinics, and by word-of-mouth. We also routinely conduct recruitment activities at government agencies such as social welfare grant offices.

Briefly, main eligibility criteria were similar for all studies, and included: being sexually active; HIV-negative status at screening; willingness to provide written consent and follow study procedure; not pregnant with no intention to become so; and anticipated residence in and around the study area for a minimum of 1 year. Participants received risk reduction counselling and access to condoms at all visits. Counsellors emphasized that condoms are the only known method to prevent acquisition of HIV and STIs, and that condoms should be used for every sex act. While the MIRA trial included an investigational arm which women who enrolled may have been randomised to, and which may have influenced their willingness to participate, both HPTN 055 and the MDP Feasibility study were cohort studies conducted in preparation for later trials, and women were only requested to use condoms in these latter two investigations.

\section{Study Procedures}

For the MIRA and HPTN 055 trials, HIV diagnostic testing was achieved using two rapid tests on whole blood sourced from either finger-prick or venepuncture (Determine HIV1/2 Abbott Laboratories, Tokyo, Japan and Oraquick Orasure Technologies, Bethlehem, PA, USA). The Abbott IMX ELISA test (Abbott Diagnostics, Johannesburg, South Africa) in combination with the Vironostika HIV Uni-form II plus O ELISA (previously Organon Teknika, USA, currently bioMérieux, France) for positive and equivocal results was used on whole blood sourced from venepuncture for HIV diagnosis during the MDP Feasibility Study. Only women who had an HIV test result were included in the studies.

STI testing was undertaken by similar methods in all studies [16, 18]; the methods used in the MDP Feasibility Study have not previously been outlined and are presented here: Chlamydia and gonorrhoea were assessed using PCR (COBAS Amplicor, Roche Molecular Diagnostics, Pleasanton, CA, USA); syphilis by RPR and confirmatory TPHA (Omega Diagnostics, Alva, UK); HSV2 by HerpeSelect TM2 ELISA IgG (Focus Technologies, Cypress, CA,
USA); and bacterial vaginosis, Candida and T. vaginalis by Gram stain, $\mathrm{KOH}$ and Whiff test, and wet mount microscopy, respectively. Participants were treated for curable STIs prior to enrolment and assessed and treated for incident infections at each study visit.

Behavioural questionnaires to collect information such as frequency of condom use and frequency of sex acts were administered both by trained interviewers and via audio computer assisted self interview.

Women who were HIV-positive at screening were referred to local health care facilities for care and support. Data on whether seropositive women were on antiretrovirals at the time of screening were not collected. Women who seroconverted during the trials remained in the study and were provided with ongoing counselling and referral to local health care facilities for further care upon completion of the studies. All protocols and informed consent forms were approved by the Biomedical Research Ethics Committee at the University of KwaZulu-Natal as well as the various study-specific Institutional Review Boards.

\section{Statistical Analysis}

For each study/cohort, all relevant demographic, socioeconomic, sexual risk behaviors and biological factors were pooled by participants' unique identification numbers. Data were combined by variables across trials to increase the statistical power. SAS 9.2 data statements were used to combine the available individual patient-level data before the analysis was conducted. Categorical variables were created using the same cut-points across the studies. Data were not weighted since the primary concern was the comparison of characteristics by residential area. Statistical analysis was performed using STATA release 10.0 (Stata Statistical Software, Stata Corporation, College Station, Texas, TX, USA). Differences in proportions were tested for statistical significance using the Chi square test. The Student's $t$ test was used to compare continuous data. Univariate and multivariate logistic regression analyses were conducted to identify variables predictive of HIV infection at screening.

For the analyses presented here, we only considered those sociodemographic and behavioural characteristics which were assessed consistently across all three studies at screening. Variables included in this analysis were region of residence (urban, semi-rural, rural); age; marital status; relationship and cohabitation status; education level; being diagnosed with any STIs at baseline or thereafter (Chlamydia, gonorrhoea, syphilis or $T$. vaginalis) and incident pregnancy.

The incidence rate for HIV infection, expressed as time to seroconversion, was estimated for women who were HIV-negative at screening and satisfied the eligibility 
criteria. The date of seroconversion was estimated using the midpoint between the last negative and the first positive antibody test results within the follow-up period. Associations between various independent factors and HIV seroconversion were assessed with Kaplan-Meier survival plots, log-rank tests and in a multivariate model using Cox proportional hazard regression analysis. Risk factors of interest were residential area (rural or semi-rural vs. urban); age (expressed in the age groups $\leq 24,25-34$ and 35 or more); relationship and cohabitation status; education level (high school or less); condom use during last sexual act and contraception use (male condoms, natural methods, pills, female condoms, injectables and surgical sterilization). Multivariate models were created that included all variables found to be statistically significant $(P<0.1)$ in univariate analyses and used forward stepwise methods.

\section{Results}

Overall socio-demographic characteristics of the study population are presented, as well as by rural, semi-rural and urban residence in Table 1 . Of the 5,753 women who consented to screening for HIV status, 3,994 (70\%) were from a rural region, 1,226 (21\%) from a semi-rural region, and $533(9 \%)$ from an urban region. Overall the combined study population had a median age of 27 years (interquartile range (IQR): 22-34). Most of the women were single and non-cohabiting (87\%). Approximately $40 \%$ of the women had less than a high school education. The total number of person years that women were at risk was
3171.96. The retention rates in the studies were $94 \%$ (MIRA), 97 and 94\% (HPTN 055 at Durban and Hlabisa, respectively), and 67\% (MDP Feasibility study).

\section{Prevalence of HIV Infection at Screening}

A total of 2,369 participants $(43 \%)$ were HIV-positive at screening (Table 1). HIV infection at screening differed significantly by area of residence $(P=0.0002)$. The proportion of women who were aged 35 years or older was highest in rural and semi-rural areas compared to urban areas. Having less than high school education was highest among rural residents compared to semi-rural and urban. Rural and semi-rural residents were more likely than urban residents to be single and non-cohabiting (marital status was self-reported).

HIV prevalence for the age groups $\leq 24,25-34$, and $35+$ years was 38, 52 and $30 \%$, respectively.

Table 2 presents the results of univariate and multivariate logistic regression analyses of characteristics of women who tested positive for HIV infection at screening. Living in a semi-rural residential area, being between the ages of 25 and 34 years, single and non-cohabiting, and being diagnosed with any STIs at baseline (Chlamydia, gonorrhoea, syphilis or $T$. vaginalis) were all significant independent predictors of HIV infection in multivariate analysis.

\section{Incidence of HIV Seroconversion During Follow-Up}

A total of 2,523 HIV-negative women were eligible and enrolled in the three studies, with a median duration of

Table 1 Demographic characteristics, HIV and STI prevalence at screening in three communities of women from different geographical areas and overall

\begin{tabular}{|c|c|c|c|c|c|}
\hline Variables & Overall $N(\%)$ & $\operatorname{Urban}^{\mathrm{a}} N(\%)$ & $\operatorname{Rural}^{\mathrm{b}} N(\%)$ & Semi-rural $^{\mathrm{c}} N(\%)$ & $Z$ value \\
\hline Number of women $(\%)$ & $5,753(100)$ & $533(9)$ & $3,994(70)$ & $1,226(21)$ & \\
\hline $\mathrm{HIV}$ at screening & $2,369(43)$ & $190(40)$ & $1,605(41)$ & $574(47)$ & $2.72 * *$ \\
\hline $\mathrm{STI}^{\mathrm{d}}$ at screening & $788(14)$ & $132(27)$ & $620(16)$ & $70(6)$ & $5.63 * *$ \\
\hline Age, median (IQR) & $27(22-34)$ & $26(22-30)$ & $27(22-34)$ & $28(23-36)$ & \\
\hline Age groups (years) & & & & & $6.75 * *$ \\
\hline$\leq 24$ & $2,214(38)$ & $226(42)$ & $1,598(40)$ & $390(32)$ & \\
\hline $25-34$ & $2,181(38)$ & $238(45)$ & $1,456(36)$ & $487(40)$ & \\
\hline $35+$ & $1,358(24)$ & $69(13)$ & $939(24)$ & $350(28)$ & \\
\hline \multicolumn{6}{|l|}{ Education: } \\
\hline Less than high school & $2,382(41)$ & $137(26)$ & $1,912(47)$ & $334(27)$ & $7.14 * *$ \\
\hline Single, non-cohabiting & $5,033(87)$ & $434(81)$ & $3,461(87)$ & $1,138(93)$ & $3.98 * *$ \\
\hline
\end{tabular}

\footnotetext{
${ }^{a}$ Urban Durban only (not all screened participants tested for HIV $(n=481)$ or STI $\left.(n=484)\right)$

b Rural districts of Hlabisa (number of patients tested for HIV = 435), Umkomaas and Botha's Hill (southern and western Durban)

c Tongaat and Verulam (northern Durban)

d Chlamydia, gonorrhoea, syphilis or T. vaginalis

** $P$ values $<0.01$
} 
Table 2 Risk factors for being diagnosed with HIV infection at screening

\begin{tabular}{|c|c|c|c|c|}
\hline \multirow[t]{2}{*}{ Variable } & \multicolumn{2}{|l|}{ Univariate analysis } & \multicolumn{2}{|l|}{ Multivariate analysis } \\
\hline & Odds ratio $(95 \% \mathrm{CI})$ & $z$ value & Odds ratio $(95 \% \mathrm{CI})$ & $z$ value \\
\hline \multicolumn{5}{|l|}{ District } \\
\hline Urban & 1 & & 1 & - \\
\hline Rural & $1.14(0.95,1.38)$ & 1.41 & $1.15(0.94,1.40)$ & 1.33 \\
\hline Semi-rural & $1.46(1.19,1.80)$ & $2.60 * *$ & $1.36(1.10,1.70)$ & $2.31 * *$ \\
\hline $\mathrm{STI}^{\mathrm{a}}$ at screening & $1.56(1.34-1.83)$ & $2.85^{* *}$ & $1.27(1.08-1.48)$ & $2.25^{* *}$ \\
\hline \multicolumn{5}{|l|}{ Age groups (years) } \\
\hline $35+$ & 1 & - & - & \\
\hline $25-34$ & $2.62(2.27,3.03)$ & $3.62 * *$ & $2.09(1.79,2.44)$ & $2.52 * *$ \\
\hline$\leq 24$ & $1.41(1.22,1.63)$ & $1.23 * *$ & $0.97(0.83,1.13)$ & -0.31 \\
\hline Less than high school education & $0.99(0.90,1.10)$ & -0.21 & - & - \\
\hline Single, non-cohabiting & $6.11(4.86,7.70)$ & $5.39 * *$ & $6.07(4.78,7.70)$ & $5.31 * *$ \\
\hline
\end{tabular}

follow-up of 12 months. Of these, a total of 211 women seroconverted during the follow-up period (August 2002September 2005) (incidence rate 6.6/100 women-years). The Kaplan-Meier survival curves of HIV infection stratified by age and cohabitation status with the woman's regular sexual partners are shown in Fig. 1a and b respectively. The crude incidence rate was highest in women 24 years of age or younger (10/100 women-years), and second highest among single, non-cohabiting woman (6.4/100 women-years). The incidence rates in women aged 25-34 and 35+ years were 6/100 women-years and 3.6/100 women-years, respectively.

Table 3 presents the results of univariate and multivariate Cox proportional hazard analyses of demographic characteristics and their association with time to HIV seroconversion. Univariate analyses showed that baseline STI infection (Chlamydia, gonorrhoea, syphilis or T. vaginalis) (hazard ratio (HR) 1.52, 95\% confidence Interval (CI) 1.07, 2.15), incident STIs (HR 2.17, 95\% CI 1.66, 2.84), incident pregnancy (HR 2.07, 95\% CI 1.58, 2.73), being aged 25-34 (HR 1.69, 95\% CI 1.10, 2.60), being 24 years of age or younger (HR 2.78, 95\% CI 1.88, 4.11), being a single, noncohabiting woman (HR 4.76, 95\% CI 2.60, 8.74), and having used a condom at the last sex act (baseline) (HR $1.47,95 \%$ CI 1.12, 1.96), were all significantly associated with increased risk of HIV seroconversion. No significant associations with time to seroconversion were observed for geographical area, baseline education level, employment status, baseline contraception use, and number of sex acts in the week before seroconversion.

At the multivariate level, incident STIs (HR 1.90, 95\% CI 1.45, 2.50), being $\leq 24$ years old (HR 1.85, 95\% CI $1.24,2.77)$, and being a single, non-cohabiting woman (HR
$3.43,95 \%$ CI $1.83,6.42)$, were all significantly associated with increased risk of HIV seroconversion.

\section{Discussion}

The combined data from these three studies clearly suggest a very generalized and severe epidemic of HIV among a select population of non-pregnant women in the greater Durban region of KwaZulu-Natal, South Africa.

The overall incidence of HIV infection was unacceptably high at 6.6/100 woman years-almost double the incidence for non-pregnant women calculated from a country-wide prevalence survey conducted during a similar time period $(3.8 \%)$ [33]. The incidence rate was highest among women 24 years of age or younger, in contrast to the peak of prevalent HIV infection, which was found among women aged 25-35 years, a pattern also noted by Rehle et al. [34] for data gathered between 2002 and 2005. We believe this difference may be a reflection of the slow uptake of HIV testing in these populations-older women may have had undetected chronic infections of several years duration, with the date of seroconversion occurring when they were probably under the age of 24 years. Other authors have found that many women older than 25 years of age were undergoing HIV testing for the first time during screening for clinical trials [35]. Rehle et al. [34] consider that the roll-out of antiretroviral therapy has resulted in an "excess" of prevalent HIV infections with a peak of excess infections in women aged 25 years and older, which overlaps with our findings. However, this is unlikely to be the primary explanation for the high HIV prevalence in the 25-35 year age group, since roll-out of 


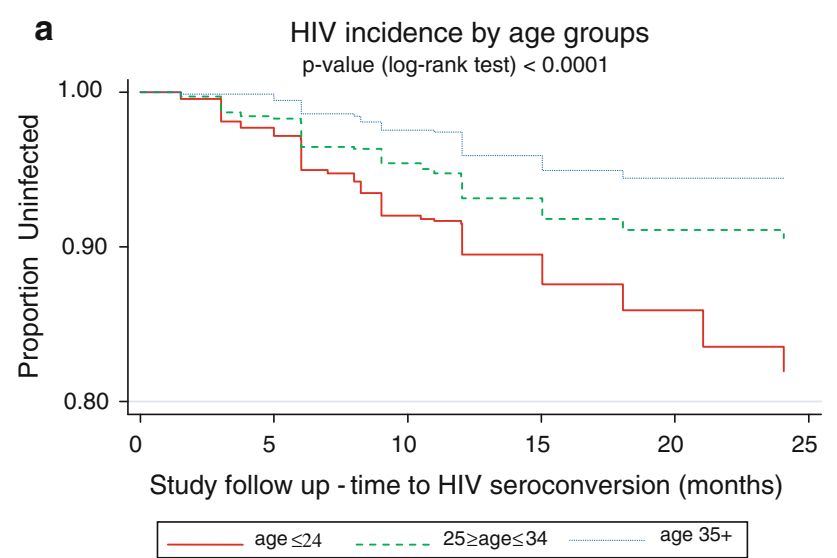

Test statistic for log rank test: 38.01

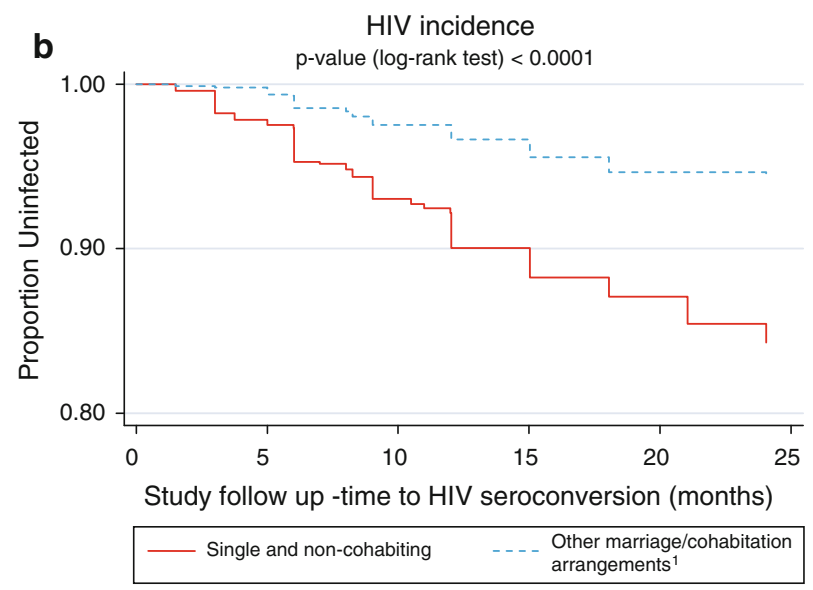

${ }^{1}$ All other marriage and cohabitation combinations e.g. married and cohabiting, married but non-cohabiting, single and cohabiting

Test statistic for log rank test: 39.44

Fig. 1 The Kaplan-Meier survival curves of HIV infection stratified by age (a) and cohabitation status (b)

ARVs for treatment only began in 2004 [36] in South Africa and the studies included in this analysis collectively spanned a time period from 2002 to 2005 .

A tentative comparison with the incidence rates for the period 2002-2005 calculated by Rehle and colleagues [34] may be made: those authors calculated a nation-wide incidence rate of 5.5/100 person years for women aged 15-24 years from national HIV survey data, which is only approximately half that found for women aged 24 years or younger in our combined cohort (10/100 woman-years). Directly measured incidence data are available from a study conducted by the Africa Centre in Hlabisa spanning 2003-2005 [37], which showed that the highest incidence of HIV occurred among women 25-29 years of age (crude incidence rate 8/100 wy), while women 20-24 years of age had a crude incidence rate of 5.6/100 woman years; total incidence for women between the ages of 15 and 49 years was 3.8/100 woman years. Similarly, Abdool Karim et al.
[35] found the highest overall HIV incidence rate among women 25 years of age or older (8.7/100 wy) in two communities in KwaZulu-Natal (one rural and one urban), while our data show peak HIV incidence among women 24 years of age or younger. While differences in the study methods and age categorisation complicate the comparison, it is clear that the HIV incidence in women in their twenties is extremely high in these studies. The recently completed CAPRISA 004 trial [19] also enables comparison of HIV incidence rates in the placebo arm with our data for similar geographic locations; the overall HIV incidence rate in the placebo arm was 9.1/100 woman years for the years 2007-2010, slightly higher than our overall figure (6.6/100 wy) for an earlier measurement period. Those authors also did not find a significant difference in incidence rates between rural and urban sites (for either active or placebo arms), in agreement with our results which indicate no significant difference in risk of seroconversion by residential area (urban or rural). Taken together, these data indicate the seriousness of the epidemic in KwaZulu-Natal and the potential future burden on health-care resources in that province as a result of HIV-related morbidity.

Risk of HIV seroconversion was also higher among women who used condoms (at last sexual act-baseline measurement) in comparison to women who did not. This apparently counterintuitive finding could be explained by patterns of sexual risk-taking in those who use condoms; condom usage has been found to be more common among men who have several casual partners [13], and usage with regular partners is unusual [38], indicating that measures of condom use may possibly be considered proxies for the level of risky sexual behaviour in sub-Saharan Africa i.e. persons who perceive themselves to be at high risk are more likely to both use condoms and be at risk of acquiring HIV.

The incidence of HIV seroconversion was higher among women who were single and non-cohabiting. We chose to explore this demographic in particular to illustrate the comparative risk of HIV acquisition in association with relationship status. We would hypothesise that the young, single, non-cohabiting women in our studies may have been involved in sexual contacts which were characterised by power asymmetries (e.g. transactional and/or intergenerational sex $[3,39,40])$; this could be the reason why these women were more vulnerable to HIV acquisition. In addition, these women may have been involved in multiple concurrent relationships [6], or long-term established noncohabiting relationships in which condom use may have decreased due to increased trust of the partner [41], both of which could increase their vulnerability to HIV.

Our data did not confirm a higher risk of prevalent HIV in urban populations, even though this group had the highest proportion of 25-34 year-olds - the age group most 
Table 3 Risk factors for HIV seroconversion during follow-up (analysis by Cox proportional hazard regression)

\begin{tabular}{|c|c|c|c|c|c|}
\hline \multirow[t]{2}{*}{ Variable } & \multirow[b]{2}{*}{$N(\%)$} & \multicolumn{2}{|l|}{ Univariate analysis } & \multicolumn{2}{|c|}{ Multivariate analysis } \\
\hline & & HR $(95 \% \mathrm{CI})$ & $z$ value & HR $(95 \% \mathrm{CI})$ & $z$ value \\
\hline Any $\mathrm{STI}^{\mathrm{a}}$ at screening & $316(13)$ & $1.52(1.07,2.15)$ & $2.80 *$ & - & - \\
\hline $\begin{array}{l}\text { Any STI }{ }^{\mathrm{a}} \text { during the follow-up } \\
\text { (time dependent) }\end{array}$ & $711(28)$ & $2.17(1.66,2.84)$ & $4.80 * *$ & $1.90(1.45,2.50)$ & $3.90 * *$ \\
\hline $\begin{array}{l}\text { Pregnancy during the follow-up } \\
\text { (time-dependent) }\end{array}$ & $566(22)$ & $2.07(1.58,2.73)$ & $3.85^{* *}$ & - & \\
\hline \multicolumn{6}{|l|}{ Age (years) } \\
\hline $35+$ & $784(31)$ & 1 & & 1 & \\
\hline $25-34$ & $774(31)$ & $1.69(1.10,2.60)$ & $2.80 *$ & $1.39(0.90,2.15)$ & 1.45 \\
\hline$\leq 24$ & $965(38)$ & $2.78(1.88,4.11)$ & $3.80 * *$ & $1.85(1.24,2.77)$ & $3.03 * *$ \\
\hline \multicolumn{6}{|l|}{ District } \\
\hline Urban & $238(10)$ & 1 & & & \\
\hline Rural & $1,720(68)$ & $1.15(0.63,2.10)$ & 0.75 & - & - \\
\hline Semi-rural & $565(22)$ & $1.39(0.73,2.66)$ & 1.37 & - & - \\
\hline \multicolumn{6}{|l|}{ Less than high school education } \\
\hline Yes & $1,674(66)$ & $0.86(0.64,1.14)$ & $0.300(-1.43)$ & - & \\
\hline No & $849(34)$ & 1 & - & - & \\
\hline \multicolumn{6}{|l|}{ Employed/income } \\
\hline No & $2,000(79)$ & $1.47(1.01,2.14)$ & $2.04 *$ & & \\
\hline Yes & $523(21)$ & 1 & & - & - \\
\hline \multicolumn{6}{|l|}{ Single and non-cohabiting? } \\
\hline $\mathrm{No}^{\mathrm{b}}$ & $1,958(78)$ & 1 & & 1 & - \\
\hline Yes & $565(22)$ & $4.76(2.60,8.74)$ & $4.41 * *$ & $3.43(1.83,6.42)$ & $3.70^{* *}$ \\
\hline \multicolumn{6}{|c|}{ Condom used during last sexual act (baseline) } \\
\hline No & $1,219(48)$ & 1 & & - & - \\
\hline Yes & $1,304(52)$ & $1.47(1.12,1.96)$ & $2.31 * *$ & - & - \\
\hline \multicolumn{6}{|l|}{ Contraception use at baseline ${ }^{c}$} \\
\hline No & $1,012(40)$ & 1 & & - & - \\
\hline Yes & $1,511(60)$ & $0.95(0.71,1.26)$ & -0.26 & - & - \\
\hline \multicolumn{6}{|c|}{$\begin{array}{l}\text { Typical number of sex acts in week } \\
\text { before seroconversion }\end{array}$} \\
\hline Less than 3 acts & 991 (39) & 1 & & & \\
\hline 3 plus acts & $1,532(61)$ & $1.35(1.01,1.82)$ & $2.08 *$ & - & \\
\hline
\end{tabular}

a Chlamydia, gonorrhoea, syphilis or T. vaginalis

b All other marriage and cohabitation combinations e.g. married and cohabiting, married and non-cohabiting, single and cohabiting

${ }^{c}$ Male condoms, natural methods, pills, female condoms, injectables and surgical sterilisation

$* P$ value $<0.05$

** $P$ value $<0.01$

at risk of being HIV positive at screening. This is in contrast to the findings of Abdool Karim et al. [35] who found a significant difference in HIV prevalence between cohorts of women living in rural and urban areas; those authors did not, however, note a significant overall difference in HIV incidence between the two locales. In addition to the 25-34 year age group, those living in a semi-rural area, those who were single and non-cohabiting, and those diagnosed with an STI such as Chlamydia, gonorrhoea, syphilis or T. vaginalis were most likely to be HIV infected at baseline.

Level of education did not appear to have any effect on risk of HIV infection either at screening or during follow-up. Earlier research in Africa suggested that higher levels of education and employment were correlated with greater risk of HIV infection, and that the association was more pronounced in rural areas [42]. However, this pattern has changed over time. Lurie et al. [43] showed that 
HIV-infected adults in South Africa with a higher education level were more likely to use condoms with regular partners. The same study also suggested that school enrolment was strongly associated with protective sexual behaviour, with correspondingly lower HIV infection. The lack of a significant association of HIV prevalence and incidence with educational level in our studies may suggest that the disease has become so widespread in the study population that this distinction has fallen away.

Women's sexual negotiating power is compromised in transactional sexual relationships [44], and promoted by economic independence [45]. However we found that employment or receipt of regular income did not significantly reduce the risk of HIV acquisition.

The relationship between incident STIs and increased risk of HIV seroconversion [46] was confirmed in this study (for Chlamydia, gonorrhoea, syphilis or $T$. vaginalis). These findings underscore the association of younger age with both HIV and STIs, and reinforce the need for improved sexual health and behavioural counselling services to facilitate early detection and treatment of STIs, and to promote safer sexual practices to prevent later acquisition of HIV.

Our analyses are unique in that we were able to combine several databases from multiple studies to provide a more in-depth understanding of the HIV epidemic in KwaZuluNatal, the epicentre of the HIV epidemic in South Africa. Our results underscore an urgent need for interventions that specifically target young, single, non-cohabiting women; these include the development of new female-initiated HIV prevention options, women empowerment initiatives, and aggressive promotion of female condoms. In addition, emphasis should be placed on education of both sexual partners on other prevention options (such as male circumcision). It is also important to promote faithfulness and monogamy to avoid the risk of HIV acquisition.

Our analyses have several limitations. We could not determine the characteristics of women who did not present for screening, and therefore must exercise caution in extrapolating our results to the population as a whole. However, the results, at the least, provide valuable information about the characteristics of women who volunteer for HIV prevention studies in KwaZulu-Natal. We were only able to analyse factors influencing HIV acquisition which were measured in the various studies, and this may have limited our ability to identify such factors; the independent variables included are limited. We were unable to determine if the difference in average age of the populations of screened women was due to the overall demographics of the surrounding population, or whether the differences arose from variations by area and age in willingness to be screened. Due to the variation of questions in collecting behavioural and socio-demographic data, we were unable to merge many key components of behavioural risk characteristics such as partner turnover; similarly, a common protocol was not used across all three studies, and we therefore cannot be sure that all categorizations are the same. This may have reduced the power of the analyses to detect significant associations. Given the geographical location of the study sites, we were able to target more rural women than urban women. However, no data concerning migration were included in these analyses. Lastly we were unable to collect any sexual behaviour data from male partners of the women.

In conclusion, this study provides a valuable direct measure of the high incidence of HIV among non-pregnant women participating in HIV prevention studies in multiple communities in KwaZulu-Natal. We provide clear evidence that young, single, non-cohabiting women are most likely to acquire HIV and urge the authorities to provide a rapid response by scaling up HIV prevention, treatment and care efforts in all these communities.

Acknowledgments We gratefully acknowledge the contribution of the women who participated in these studies. For the MIRA study, we thank the team at the University of California (San Francisco), Ibis Reproductive Health and members of the HIV Prevention Research Unit (MRC South Africa). For the HPTN study, we thank Family Health International, the Division of AIDS, the Statistical Center for HIV/AIDS Research and Prevention (SCHARP), and the HIV Prevention Research Unit in Durban and Hlabisa. For the MDP Feasibility Study we thank MRC Clinical Trials Unit UK, Imperial College, and the South African MRC in Tongaat and Verulam. We would particularly like to extend our thanks to Nicola Coumi, Sharika Gappoo and Neetha Morar of the South African MRC. We acknowledge the financial support of the UK Medical Research Council, the UK Department for International Development (MDP Feasibility study: Grant number G0100137), the Bill and Melinda Gates Foundation (MIRA: Grant number 21082) and the Division of AIDS of the National Institutes of Health, USA (HPTN 055: Grant U01 AI048008).

Open Access This article is distributed under the terms of the Creative Commons Attribution Noncommercial License which permits any noncommercial use, distribution, and reproduction in any medium, provided the original author(s) and source are credited.

\section{References}

1. UNAIDS. Report on the global AIDS epidemic. Geneva: Joint United Nations Programme on HIV/AIDS; 2008.

2. Chen L, Jha P, Stirling B, et al. Sexual risk factors for HIV infection in early and advanced HIV epidemics in sub-Saharan Africa: systematic overview of 68 epidemiological studies. PLoS One. 2007;2(10):e1001.

3. Dunkle K. Transactional sex among women in Soweto, South Africa: prevalence, risk factors and association with HIV infection. Soc Sci Med. 2004;59(8):1581-92.

4. Côté A, Sobela F, Dzokoto A, et al. Transactional sex is the driving force in the dynamics of HIV in Accra, Ghana. AIDS. 2004;18(6):917.

5. Epstein H. AIDS and the irrational. BMJ. 2008;337:a2638.

6. Halperin D, Epstein H. Why is HIV prevalence so severe in southern Africa? The role of multiple concurrent partnerships and 
lack of male circumcision: implications for AIDS prevention. South Afr J HIV Med. 2007;8:19-25.

7. Cohen M, Hoffman I, Royce R, et al. Reduction of concentration of HIV-1 in semen after treatment of urethritis: implications for prevention of sexual transmission of HIV-1. Lancet. 1997; 349(9069):1868-73.

8. Ramjee G, Williams B, Gouws E, Van Dyck E, Deken B, Karim $\mathrm{S}$. The impact of incident and prevalent herpes simplex virus-2 infection on the incidence of HIV-1 infection among commercial sex workers in South Africa. J Acquir Immune Defic Syndr. 2005;39(3):333

9. Weiss H, Quigley M, Hayes R. Male circumcision and risk of HIV infection in sub-Saharan Africa: a systematic review and meta-analysis. AIDS. 2000;14(15):2361.

10. Siegfried N, Muller M, Deeks J, et al. HIV and male circumcision-a systematic review with assessment of the quality of studies. Lancet Infect Dis. 2005;5(3):165-73.

11. Coovadia H, Jewkes R, Barron P, Sanders D, McIntyre D. The health and health system of South Africa: historical roots of current public health challenges. Lancet. 2009;374(9692):817-34.

12. Lurie M, Williams B, Zuma K, et al. Who infects whom? HIV-1 concordance and discordance among migrant and non-migrant couples in South Africa. AIDS. 2003;17(15):2245-52.

13. Lurie M, Williams B, Zuma K, et al. The impact of migration on HIV-1 transmission in South Africa: a study of migrant and nonmigrant men and their partners. Sex Transm Dis. 2003; 30(2):149-56.

14. Shisana O, Rehle T, Simbayi LC, et al. South African national HIV prevalence, incidence, behaviour and communication survey 2008: a turning tide among teenagers?. Cape Town: HSRC Press; 2009.

15. Department of Health. National antenatal sentinel HIV and syphilis prevalence survey, South Africa. Pretoria: National Department of Health; 2008.

16. Ramjee G, Kapiga S, Weiss $S$, et al. The value of site preparedness studies for future implementation of phase $2 / \mathrm{IIb} / \mathrm{III}$ HIV prevention trials-experience from the HPTN 055 study. J Acquir Immune Defic Syndr. 2008;47(1):93-100.

17. Shisana O, Rehle T, Simbayi LC, et al. South African national HIV prevalence, HIV incidence, behaviour and communication survey 2005. Cape Town: HSRC Press; 2005.

18. Padian N, van der Straten A, Ramjee G, et al. Diaphragm and lubricant gel for prevention of HIV acquisition in southern African women: a randomised controlled trial. Lancet. 2007; 370(9583):251-61.

19. Abdool Karim Q, Abdool Karim SS, Frohlich JA, et al. Effectiveness and safety of tenofovir gel, an antiretroviral microbicide, for the prevention of HIV infection in women. Science. 2010;329(5996):1168-74.

20. van Damme L, Ramjee G, Alary M, et al. Effectiveness of COL1492, a nonoxynol-9 vaginal gel, on HIV-1 transmission in female sex workers: a randomised controlled trial. Lancet. 2002;360(9338):971-7.

21. Skoler-Karpoff S, Ramjee G, Ahmed K, et al. Efficacy of carraguard for prevention of HIV infection in women in South Africa: a randomised, double-blind, placebo-controlled trial. Lancet. 2008;372(9654):1977-87.

22. Feldblum P, Adeiga A, Bakare R, et al. SAVVY vaginal gel (C31G) for prevention of HIV infection: a randomized controlled trial in Nigeria. PLoS One. 2008;3(1):e1471.

23. van Damme L, Govinden R, Mirembe F, et al. Lack of effectiveness of cellulose sulfate gel for the prevention of vaginal HIV transmission. N Engl J Med. 2008;359(5):463-72.

24. Abdool Karim S, Coletti A, Richardson B, et al. Safety and effectiveness of vaginal microbicides BufferGel and $0.5 \%$ PRO 2000 gel for the prevention of HIV infection in women: results of the HPTN 035 trial [Abstract 48LB]. 16th Conference on Retroviruses and Opportunistic Infections. Montréal, 8-11 February, 2009.

25. McCormack S, Ramjee G, Kamali A, et al. PRO2000 vaginal gel for prevention of HIV-1 infection (Microbicides Development Programme 301): a phase 3, randomised, double-blind, parallelgroup trial. Lancet. 2010;376(9749):1329-37.

26. Parekh BS, Kennedy MS, Dobbs T, et al. Quantitative detection of increasing HIV type 1 antibodies after seroconversion: a simple assay for detecting recent HIV infection and estimating incidence. AIDS Res Hum Retroviruses. 2002;18(4):295-307.

27. Suligoi B, Massi M, Galli C, et al. Identifying recent HIV infections using the avidity index and an automated enzyme immunoassay. J Acquir Immune Defic Syndr. 2003;32(4):424-8.

28. van de Wijgert J, Braunstein S, Vyankandondera J, et al. Crosssectional estimation of HIV incidence by BED-CEIA and avidity index assays in vaginal microbicide preparedness studies in Rwanda and South Africa [Abstract no. 365]. Pittsburgh: Microbicides 2010; 2010.

29. Truong H-HM, Kellogg T, Louie B, Klausner J, Dilley J, McFarland W. Recent HIV-1 infection detection: comparison of incidence estimates derived by laboratory assays and repeat testing data. J Acquir Immune Defic Syndr. 2009;51(4):502-5.

30. UNAIDS Reference Group for Estimates MaP. Statement on the use of the BED-Assay for the estimation of HIV-1 incidence for surveillance or epidemic monitoring. 2005.

31. Brookmeyer R. Measuring the HIV/AIDS epidemic: approaches and challenges. Epidemiol Rev. 2010;32(1):26-37.

32. Nunn A, McCormack S, Crook A, Pool R, Rutterford C, Hayes R. Microbicides development programme: design of a phase III trial to measure the efficacy of the vaginal microbicide PRO 2000/5 for HIV prevention. Trials. 2009;10(1):99.

33. Rehle T, Shisana O, Pillay V, Zuma K, Puren A, Parker W. National HIV incidence measures-new insights into the South African epidemic. S Afr Med J. 2007;97(3):194-9.

34. Rehle TM, Hallett TB, Shisana O, et al. A decline in new HIV infections in South Africa: estimating HIV incidence from three national HIV surveys in 2002, 2005 and 2008. PLoS One. 2010;5(6):e11094.

35. Abdool Karim Q, Kharsany AB, Frohlich JA, et al. Stabilizing HIV prevalence masks high HIV incidence rates amongst rural and urban women in KwaZulu-Natal, South Africa. Int J Epidemiol. 2010.

36. Chigwedere P, Seage GRI, Gruskin S, Lee T-H, Essex M. Estimating the lost benefits of antiretroviral drug use in South Africa. J Acquir Immune Defic Syndr. 2008;49(4):410-5.

37. Bärnighausen T, Tanser F, Gqwede Z, Mbizana C, Herbst K, Newell M-L. High HIV incidence in a community with high HIV prevalence in rural South Africa: findings from a prospective population-based study. AIDS. 2008;22(1):139-44.

38. Maharaj P, Cleland J. Condom use within marital and cohabiting partnerships in KwaZulu-Natal, South Africa. Stud Fam Plann. 2004;35(2):116-24.

39. Luke N. Age and economic asymmetries in the sexual relationships of adolescent girls in sub-Saharan Africa. Stud Fam Plann. 2003;34(2):67-86

40. Hunter M. The materiality of sex: thinking beyond prostitution. Afr Stud. 2002;61(1):99-120.

41. Maharaj P. Patterns of condom use: perspectives of men in KwaZulu-Natal, South Africa. Dev South Afr. 2005;22(2): 187-97.

42. Hargreaves J, Glynn J. Educational attainment and HIV-1 infection in developing countries: a systematic review. Trop Med Int Health. 2002;7(6):489-98.

43. Lurie M, Pronyk P, de Moor E, et al. Sexual behavior and reproductive health among HIV-infected patients in urban and 
rural South Africa. J Acquir Immune Defic Syndr. 2008;47(4): 484-93.

44. Laga M, Schwärtlander B, Pisani E, Sow PS, Caraël M. To stem HIV in Africa, prevent transmission to young women. AIDS. 2001;15:931-4.

45. Greig F, Koopman C. Multilevel analysis of women's empowerment and HIV prevention: quantitative survey results from a preliminary study in Botswana. AIDS Behav. 2003;7(2): 195-208.

46. Fleming DT, Wasserheit JN. From epidemiological synergy to public health policy and practice: the contribution of other sexually transmitted diseases to sexual transmission of HIV infection. Sex Transm Infect. 1999;75(1):3-17. 